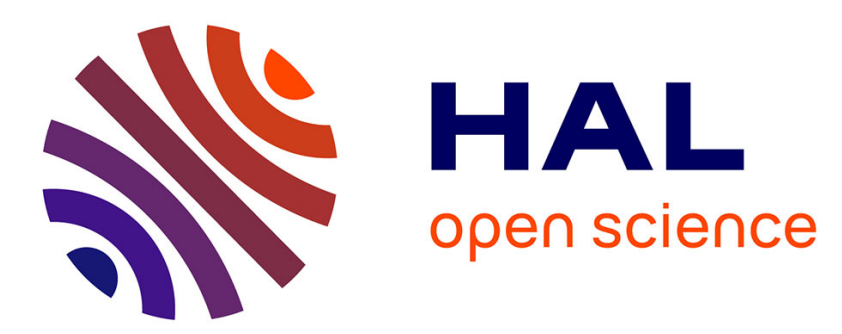

\title{
A generalized formulation of the Linear Sampling Method with exact characterization of targets in terms of farfield measurements
}

Lorenzo Audibert, Houssem Haddar

\section{- To cite this version: \\ Lorenzo Audibert, Houssem Haddar. A generalized formulation of the Linear Sampling Method with exact characterization of targets in terms of farfield measurements. Inverse Problems, 2014, 30 (035011), 10.1088/0266-5611/30/3/035011 . hal-00911692}

\author{
HAL Id: hal-00911692 \\ https://hal.inria.fr/hal-00911692
}

Submitted on 3 Dec 2013

HAL is a multi-disciplinary open access archive for the deposit and dissemination of scientific research documents, whether they are published or not. The documents may come from teaching and research institutions in France or abroad, or from public or private research centers.
L'archive ouverte pluridisciplinaire HAL, est destinée au dépôt et à la diffusion de documents scientifiques de niveau recherche, publiés ou non, émanant des établissements d'enseignement et de recherche français ou étrangers, des laboratoires publics ou privés. 


\title{
A generalized formulation of the Linear Sampling Method with exact characterization of targets in terms of farfield measurements
}

\author{
Lorenzo Audibert ${ }^{\sharp \dagger} \quad$ Houssem Haddar ${ }^{\sharp}$ \\ \#INRIA Saclay Ile de France and Ecole Polytechnique (CMAP) Route de Saclay, \\ 91128, Palaiseau, France. \\ ${ }^{\dagger}$ EDF R\&D, STEP department, Chatou, France \\ E-mail: Lorenzo.Audibert@edf.fr, Houssem.Haddar@inria.fr
}

\begin{abstract}
We propose and analyze a new formulation of the Linear Sampling Method that uses an exact characterization of the targets shape in terms of the so-called farfield operator (at a fixed frequency). This characterization is based on constructing nearby solutions of the farfield equation using minimizing sequences of a least squares cost functional with an appropriate penalty term. We first provide a general framework for the theoretical foundation of the method in the case of noise-free and noisy measurements operator. We then explicit applications for the case of inhomogeneous inclusions and indicate possible straightforward generalizations. We finally validate the method through some numerical tests and compare the performances with classical LSM and the factorization methods.
\end{abstract}

AMS classification scheme numbers: 35R60, 35R30, 65M32

Keywords: Inverse scattering problems, Linear Sampling Method, Factorization Method, Qualitative methods

\section{Introduction}

This work can be seen as a contribution to the development of so-called qualitative methods $[8,13,4]$ for solving inverse scattering problems for extended targets from fixed frequency multi-static data. More specifically, we introduce and analyze a new formulation of the so-called Linear Sampling Method (LSM) [7, 6], that we will refer to as Generalized Linear Sampling Method (GLSM), which is based on an new exact characterization of the targets shape in terms of the so-called farfield operator (at a fixed frequency). This characterization is based on constructing nearby solutions to the farfield equation as minimizing sequences of a special cost functional and uses two (complementary) factorizations of the farfield operator. The first one is the basic factorization used in the theoretical justification behind LSM and the second one is the one used by the factorization method (FM) [12, 13]. This combination allows us for instance to require less restrictive assumptions than FM. It also turns out that one can establish a direct link between our method and FM for a special setting of GLSM 
and this also provides a direct link with the analysis in [1, 2] justifying the use of LSM in some particular configurations. Although not directly inspired by them, the GLSM share some similarities with the so-called inf-criterion [13] or the formulation of this criterion in [14] as well as the probe method [11,9].

The main idea behind our method is to explicitly construct the nearby solution of the LSM by adding to a standard least squares misfit functional a penalty term proportional to an appropriate norm of the associated Herglotz wave. Using the second factorization of the farfield operator (as used in FM), we express this term using the measured farfield operator. The main issues to address are first how to cope with the fact that the penalty term is compact and second how to address the case of noisy operators. Here comes the role of the first factorization generally used for LSM. For more details we refer to the third section where the general formulation of the method is presented as well as the analysis for different configurations. In order to introduce the main ideas behind GLSM as well as a concrete application we choose to present the case of scalar inverse scattering form inhomogeneous inclusions. We show for this example how the method can be applied and we also indicate other possible straightforward applications (which are roughly speaking all cases where FM applies, or more generally where the inf-criterion and LSM apply).

The impact of our method on the numerical side is twofold. In fact, the analysis of GLSM for noisy farfield operators suggests a different indicator function for LSM than the one usually used. This new indicator function is similar to the one proposed in [1] but contains an additional term that correctly fix the behavior of the indicator function outside the obstacle for noisy operators. The superiority of this new indicator function is demonstrated through some numerical results. The second alternative is to directly use the minimizing sequence constructed by GLSM, which is computationally more expensive but leads to better results for multi-connected objects. In fact the second numerical method can be used as a post-processing of the first one since from numerical experiments, we observed that only few iterations are needed to update the initial guess provided by LSM.

The article is organized as follows. In Section 2 a model problem is introduced to motivate GLSM after recalling the basis of the LSM and the factorization method. The theoretical foundation of the GLSM is given in Section 3. Section 4 provides an example of application of GLSM by completely treating the model problem introduced in Section 2 and indicating other possible applications. The last section (Section 5) is devoted to the introduction of two numerical algorithms issued from Section 3 along with validating numerical results and comparison with other algorithms.

\section{A model problem and motivation for GLSM}

In order to introduce the ideas and motivations behind the proposed new algorithm below, namely GLSM, we choose to present as a model problem the scalar inverse time harmonic scattering problem from inhomogenous targets. For a wave number $k>0$, 
the total field solves the Helmholtz equation

$$
\Delta u+k^{2} n u=0 \text { in } \mathbb{R}^{d}
$$

with $d=2$ or 3 and with $n \in L^{\infty}\left(\mathbb{R}^{d}\right)$ denoting the refractive index such that the support of $n-1$ is equal to $\bar{D}$ with $D$ a bounded domain with Lipschitz boundary and connected complement and such that $\Im(n) \geq 0$. We are interested in the cases where the total field is generated by plane waves, $u^{i}(\theta, x):=e^{i k x \cdot \theta}$ with $x \in \mathbb{R}^{d}$ and $\theta \in \mathbb{S}^{d-1}$ (the unit sphere) and we denote by $u^{s}$ the scattered field defined by

$$
u^{s}(\theta, \cdot)=u-u^{i}(\theta, \cdot) \quad \text { in } \mathbb{R}^{d}
$$

which is assumed to be satisfying the Sommerfeld radiation condition,

$$
\lim _{r \rightarrow \infty} \int_{|x|=r}\left|\frac{\partial u^{s}}{\partial r}-i k u^{s}\right|^{2} d s=0 .
$$

Our data for the inverse problem will be formed by noisy measurements of so called farfield pattern $u^{\infty}(\theta, \hat{x})$ defined by

$$
u^{s}(\theta, x)=\frac{e^{i k|x|}}{|x|^{(d-1) / 2}}\left(u^{\infty}(\theta, \hat{x})+O(1 /|x|)\right)
$$

as $|x| \rightarrow \infty$ for all $(\theta, \hat{x}) \in \mathbb{S}^{d-1} \times \mathbb{S}^{d-1}$. The goal is to be able to reconstruct $D$ from these measurements (without knowing $n$ ) using a new sampling algorithm. The foundation of this algorithm is inspired by the Linear Sampling Method and the Factorization Method that we shall briefly review here in the context of this special scattering problem. These methods are based on the farfield operator $F: L^{2}\left(\mathbb{S}^{d-1}\right) \rightarrow L^{2}\left(\mathbb{S}^{d-1}\right)$, defined by

$$
F g(\hat{x}):=\int_{\mathbb{S}^{d-1}} u^{\infty}(\theta, \hat{x}) g(\theta) d s(\theta) .
$$

Let us define for $\psi \in L^{2}(D)$, the unique function $w \in H_{\text {loc }}^{1}\left(\mathbb{R}^{d}\right)$ satisfying

$$
\left\{\begin{array}{l}
\Delta w+n k^{2} w=k^{2}(1-n) \psi \text { in } \mathbb{R}^{d}, \\
\lim _{r \rightarrow \infty} \int_{|x|=r}\left|\frac{\partial w}{\partial r}-i k w\right|^{2} d s=0 .
\end{array}\right.
$$

By linearity of the forward scattering problem, $F g$ is nothing but the farfield pattern of $w$ solution of $(1)$ with $\psi=v_{g}$ in $D$, where

$$
v_{g}(x):=\int_{\mathbb{S}^{d-1}} e^{i k x \cdot \theta} g(\theta) d s(\theta), g \in L^{2}\left(\mathbb{S}^{d-1}\right), x \in \mathbb{R}^{d} .
$$

Now consider the (compact) operator $H: L^{2}\left(\mathbb{S}^{d-1}\right) \rightarrow L^{2}(D)$ defined by

$$
H g:=\left.v_{g}\right|_{D},
$$

and the (compact) operator $G: \overline{\mathcal{R}(H)} \subset L^{2}(D) \rightarrow L^{2}\left(\mathbb{S}^{d-1}\right)$ defined by

$$
G \psi:=w^{\infty}
$$


where $w^{\infty}$ is the farfield of $w \in H_{\text {loc }}^{1}\left(\mathbb{R}^{d}\right)$ solution of $(1)$ and where $\overline{\mathcal{R}(H)}$ denotes the closure of the range of $H$ in $L^{2}(D)$. Then clearly

$$
F=G H \text {. }
$$

The basis of the Linear Sampling Method is the following characterization of $D$ in terms of the range of $G$. This characterization is based on the solvability of so called interior transmission problem defined by $(u, v) \in L^{2}(D) \times L^{2}(D)$ such that $u-v \in H^{2}(D)$ and

$$
\left\{\begin{array}{l}
\Delta u+k^{2} n u=0 \quad \text { in } D, \\
\Delta v+k^{2} v=0 \quad \text { in } D \\
(u-v)=f \quad \text { on } \partial D \\
\frac{\partial}{\partial \nu}(u-v)=g \quad \text { on } \partial D
\end{array}\right.
$$

for given $f \in H^{\frac{1}{2}}(\partial D)$ and $g \in H^{-\frac{1}{2}}(\partial D)$. We shall make the following assumption

Hypothesis 1. We assume that $k^{2} \in \mathbb{R}_{+}$and $n \in L^{\infty}(D)$ are such that, $\Im(n) \geq 0$ and such that for all $f \in H^{\frac{1}{2}}(\partial D)$ and $g \in H^{-\frac{1}{2}}(\partial D)$ problem (3) has a unique solution $(u, v) \in L^{2}(D) \times L^{2}(D)$ such that $u-v \in H^{2}(D)$.

It is well known for instance that if in addition, $1 /(n-1) \in L^{\infty}(D)$ and $\Re(n-1)$ is positive definite or negative definite in a neighborhood of $\partial D$, then Hypothesis 1 is verified for all $k \in \mathbb{R}$ except a countable set without any finite accumulation point [18]. Defining

$$
\phi_{z}(\hat{x}):=e^{-i k \hat{x} \cdot z}
$$

the main ingredient of LSM is the following.

Theorem 1. Under Hypothesis $1, \phi_{z} \in \mathcal{R}(G)$ if and only if $z \in D$.

The proof of this theorem is rather straightforward using the important result of Lemma 1 (see [15]) and the fact that $\phi_{z}$ is the farfield of $\Phi(\cdot ; z)$, the fundamental solution of the Helmholtz equation satisfying the Sommerfeld radiation condition.

Lemma 1. $\overline{\mathcal{R}(H)}=\left\{v \in L^{2}(D) ; \Delta v+k^{2} v=0\right.$ in $\left.D\right\}$.

From Theorem 1 one can deduce the following statement, which is the basic theoretical justification of the LSM.

Theorem 2. Under Hypothesis 1, the operator $F$ is injective with dense range. Moreover, the following holds.

- If $z \in D$ then there exists $g_{z}^{\epsilon}$ such that $\left\|F g_{z}^{\epsilon}-\phi_{z}\right\|_{L^{2}\left(\mathbb{S}^{d-1}\right)} \leq \epsilon$ and $\limsup _{\epsilon \rightarrow 0}\left\|H g_{z}^{\epsilon}\right\|_{L^{2}(D)}<\infty$.

- If $z \notin D$ then for all $g_{z}^{\epsilon}$ such that $\left\|F g_{z}^{\epsilon}-\phi_{z}\right\|_{L^{2}\left(\mathbb{S}^{d-1}\right)} \leq \epsilon, \lim _{\epsilon \rightarrow 0}\left\|H g_{z}^{\epsilon}\right\|_{L^{2}(D)}=\infty$.

This theorem thus suggests to use a nearby solution to $F g_{z}^{\epsilon} \simeq \phi_{z}$ for different sampling points $z$ to obtain an indicator of $D$. Two problematic issues are then raised: the first one is that the indicator function (provided by the theorem) should be $\left\|H g_{z}^{\epsilon}\right\|_{L^{2}(D)}$ which depends on $D$ and the second one is that the theorem does not 
give explicit construction of $g_{z}^{\epsilon}$. In practice, a Tikhonov regularization is usually used to build a nearby solution (as suggested by the first statement in Theorem 2) and $\left\|g_{z}^{\epsilon}\right\|_{L^{2}\left(\mathbb{S}^{d-1}\right)}$ is used in replacement of $\left\|H g_{z}^{\epsilon}\right\|_{L^{2}(D)}$. In [1] it is proved, based on the Factorization method, that Tikhonov regularization provides the good solution as soon as $\Im(n)=0$ and in that case one can replace $\left\|H g_{z}^{\epsilon}\right\|_{L^{2}(D)}$ with $\left|H g_{z}^{\epsilon}(z)\right|$. As it will be seen later, the proposed GLSM gives an alternative solution independent from the Factorization method (although inspired by this method) and more importantly that efficiently treats the case of noisy operator.

The idea behind GLSM is as simple as reconstructing a nearby solution of the LSM by using a least squares misfit functional with a penalty term that controls $\left\|H g_{z}^{\epsilon}\right\|_{L^{2}(D)}^{2}$. This is feasible thanks to the second factorization of the farfield operator, which is the starting point of the Factorization method. More precisely, for the case under consideration, since the farfield operator of $w$ has the following expression ([8])

$$
w^{\infty}(\hat{x})=-\int_{\mathcal{D}} e^{-i k y \cdot \hat{x}}(1-n) k^{2}(\psi(y)+w(y)) d y,
$$

one simply has $G=H^{*} T \psi$ where $H^{*}: L^{2}(D) \rightarrow L^{2}\left(\mathbb{S}^{d-1}\right)$ is the adjoint of $H$ given by

$$
H^{*} \varphi(\hat{x}):=\int_{D} e^{-i k y \cdot \hat{x}} \varphi(y) d y, \varphi \in L^{2}(D), \hat{x} \in \mathbb{S}^{d-1},
$$

and where $T: L^{2}(D) \rightarrow L^{2}(D)$ is defined by

$$
T \psi:=-k^{2}(1-n)(\psi+w),
$$

with $w \in H_{\text {loc }}^{1}\left(\mathbb{R}^{d}\right)$ being the solution of (1). Finally we get

$$
F=H^{*} T H,
$$

which indicates that $(F g, g)_{L^{2}\left(\mathbb{S}^{d-1}\right)}=(T(H g), H g)_{L^{2}(D)}$. Therefore, if the operator $T$ satisfies some appropriate coercivity property, the term $(F g, g)_{L^{2}\left(\mathbb{S}^{d-1}\right)}$ would be equivalent to $\left\|H g_{z}^{\epsilon}\right\|_{L^{2}(D)}^{2}$. One then can use $\left|(F g, g)_{L^{2}\left(\mathbb{S}^{d-1}\right)}\right|$ as a penalty term and also as a criterion for building the indicator function. This is the starting point of GLSM. The detailed formulation and analysis of the method are given in the next section.

\section{Theoretical Foundations of GLSM}

In this section we shall give the theoretical foundations of the Generalized Linear Sampling Method. The general framework is given by the following assumptions. We shall denote by $X$ and $Y$ two (complex) reflexive Banach spaces with duals $X^{*}$ and $Y^{*}$ respectively and shall denote by $\langle$,$\rangle a duality product that refers to \left\langle X^{*}, X\right\rangle$ or $\left\langle Y^{*}, Y\right\rangle$ duality. We consider two bounded linear operators $F: X \rightarrow X^{*}$ and $B: X \rightarrow X^{*}$ that are assumed to be bounded. Moreover we shall assume that the following factorizations hold

$$
F=G H \quad \text { and } \quad B=H^{*} T H
$$

where the operators $H: X \rightarrow Y, T: Y \rightarrow Y^{*}$ and $G: \overline{\mathcal{R}(H)} \subset Y \rightarrow X^{*}$ are bounded, where $\overline{\mathcal{R}(H)}$ the closure of the range of $H$ in $Y$. 


\subsection{Formulation of GLSM for noise free measurements}

Let $\alpha>0$ be a given parameter and $\phi \in X^{*}$. The GLSM (for noisy free measurements) is based on considering minimizing sequences of the functional $J_{\alpha}(\phi ; \cdot): X \rightarrow \mathbb{R}$

$$
J_{\alpha}(\phi ; g):=\alpha|\langle B g, g\rangle|+\|F g-\phi\|^{2} \quad \forall g \in X .
$$

Indeed this functional has not a minimizer in general. However, since $J_{\alpha}(\phi ; \cdot) \geq 0$ one can define

$$
j_{\alpha}(\phi):=\inf _{g \in X} J_{\alpha}(\phi ; g) .
$$

Then the first simple observation is the following.

Lemma 2. Assume that $F$ has dense range. Then for all $\phi \in X^{*}, j_{\alpha}(\phi) \rightarrow 0$ as $\alpha \rightarrow 0$.

Proof. Since $F$ has dense range, for a given $\epsilon>0$ there exists $g_{\epsilon}$ such that $\left\|F g_{\epsilon}-\phi\right\|<\frac{\epsilon}{2}$. Then one can choose $\alpha_{0}(\epsilon)$ such for all $\alpha \leq \alpha_{0}(\epsilon), \alpha\left|\left\langle B g_{\epsilon}, g_{\epsilon}\right\rangle\right|<\frac{\epsilon}{2}$ so that $j_{\alpha}(\phi)<\epsilon$, which proves the claim.

The central theorem for noisy free GLSM is the following characterization of the range of $G$ in terms of $F$ and $B$.

Theorem 3. We assume in addition that

- $G$ is compact and $F=G H$ has dense range.

- $T$ satisfies the coercivity property

$$
|\langle T \varphi, \varphi\rangle|>\mu\|\varphi\|^{2} \quad \forall \varphi \in \mathcal{R}(H),
$$

where $\mu>0$ is a constant independent of $\varphi$. Let $C>0$ be a given constant (independent of $\alpha$ ) and consider for $\alpha>0$ and $\phi \in X^{*}, g_{\alpha} \in X$ such that

$$
J_{\alpha}\left(\phi ; g_{\alpha}\right) \leq j_{\alpha}(\phi)+C \alpha .
$$

Then $\phi \in \mathcal{R}(G)$ if and only if $\limsup _{\alpha \rightarrow 0}\left|\left\langle B g_{\alpha}, g_{\alpha}\right\rangle\right|<\infty$ which is true if and only if $\liminf _{\alpha \rightarrow 0}\left|\left\langle B g_{\alpha}, g_{\alpha}\right\rangle\right|<\infty$.

Proof. - Assume that $\phi \in \mathcal{R}(G)$. Then, by definition one can find $\varphi \in \overline{\mathcal{R}(H)}$ such that $G \varphi=\phi$. for $\alpha>0, \exists g_{0} \in X$ such that $\left\|H g_{0}-\varphi\right\|^{2}<\alpha$. Then by continuity of $G,\left\|F g_{0}-\phi\right\|^{2}<\|G\|^{2} \alpha$. On the other hand the continuity of $T$ implies

$$
\left|\left\langle B g_{0}, g_{0}\right\rangle\right|=\left|\left\langle T H g_{0}, H g_{0}\right\rangle\right| \leq\|T\|\left\|H g_{0}\right\|^{2}<2\|T\|\left(\alpha+\|\varphi\|^{2}\right)
$$

From the definitions of $j_{\alpha}(\phi)$ and $g_{\alpha}$ we have

$$
\alpha\left|\left\langle B g_{0}, g_{0}\right\rangle\right|+\left\|F g_{0}-\phi\right\|^{2}>j_{\alpha}(\phi)>J_{\alpha}\left(\phi, g_{\alpha}\right)-C \alpha
$$

We then deduce from the definition of $J_{\alpha}$ and previous inequalities

$$
\alpha\left|\left\langle B g_{\alpha}, g_{\alpha}\right\rangle\right| \leq J_{\alpha}\left(\phi, g_{\alpha}\right) \leq C \alpha+2 \alpha\|T\|\left(\alpha+\|\varphi\|^{2}\right)+\alpha\|G\|^{2} .
$$

Therefore $\limsup _{\alpha \rightarrow 0}\left|\left\langle B g_{\alpha}, g_{\alpha}\right\rangle\right|<\infty$. This also implies $\liminf _{\alpha \rightarrow 0}\left|\left\langle B g_{\alpha}, g_{\alpha}\right\rangle\right|<\infty$. 
- Assume that $\phi \notin \mathcal{R}(G)$ and assume (by a contradiction argument) that $\liminf _{\alpha \rightarrow 0}\left|\left\langle B g_{\alpha}, g_{\alpha}\right\rangle\right|<\infty$. Then, (for some extracted subsequence $g_{\alpha}$ ) $\left|\left\langle B g_{\alpha}, g_{\alpha}\right\rangle\right|<A$ for some constant $A$ independent of $\alpha \rightarrow 0$. The coercivity of $T$ implies that $\left\|H g_{\alpha}\right\|$ is also bounded. Since $Y$ is reflexive, then one can assume that, up to an extracted subsequence, $H g_{\alpha}$ weakly converges to some $\varphi$ in $Y$. In fact $\varphi \in \overline{\mathcal{R}(H)}$ since the latter is a convex set. Since $G$ is compact, we obtain that $G H g_{\alpha}$ strongly converges to $G \varphi$ as $\alpha \rightarrow 0$. On the other hand, Lemma 2 and the definition $J_{\alpha}\left(\phi, g_{\alpha}\right)$ imply that $\left\|F g_{\alpha}-\phi\right\| \leq J_{\alpha}\left(\phi, g_{\alpha}\right) \leq j_{\alpha}(\phi)+C \alpha \rightarrow 0$ as $\alpha \rightarrow 0$. Since $F g_{\alpha}=G H g_{\alpha}$ we obtain that $G \varphi=\phi$ which is a contradiction. We then conclude that if $\phi \notin \mathcal{R}(G)$ then $\liminf _{\alpha \rightarrow 0}\left|\left\langle B g_{\alpha}, g_{\alpha}\right\rangle\right|=\infty$. The latter also implies $\limsup _{\alpha \rightarrow 0}\left|\left\langle B g_{\alpha}, g_{\alpha}\right\rangle\right|=\infty$.

As indicated in the previous section, the range of the operator $G$ characterizes the inclusion $D$. Therefore this theorem would lead to a characterization of $D$ in terms of the operators $F$ and $B$. It also stipulates that an indicator function is given by $\left|\left\langle B g_{\alpha}, g_{\alpha}\right\rangle\right|$ for small values of $\alpha$. Let us note that the parameter $\alpha$ does not play the role of a regularization parameter, since for foreseen applications, the operator $B$ is in general compact. However, constructing a sequence $\left(g_{\alpha}\right)$ satisfying (9) for fixed $\alpha>0$ may be viewed as a regularization of the minimization of $J_{\alpha}(\phi ; \cdot)$ that can be used for numerics. A different regularization procedure that would be more suited for noisy operators is introduced in the following subsection.

Let us finally remark that in most of the applications that we have in mind, taking $B=F$ would be sufficient. In this particular case one can state the following straightforward corollary.

Corollary 1. Assume that $G(\varphi)=H^{*} T(\varphi)$ for all $\varphi \in \mathcal{R}(H)$ and assume in addition that

- $H$ is compact and $F$ has dense range,

- $T$ satisfies the coercivity property (8).

Let $C>0$ be a given constant (independent of $\alpha$ ) and consider for $\alpha>0$ and $\phi \in X^{*}$, $g_{\alpha} \in X$ such that

$$
J_{\alpha}\left(\phi ; g_{\alpha}\right) \leq j_{\alpha}(\phi)+C \alpha .
$$

Then $\phi \in \mathcal{R}(G)$ if and only if $\limsup _{\alpha \rightarrow 0}\left|\left\langle F g_{\alpha}, g_{\alpha}\right\rangle\right|<\infty$ which is true if and only if $\liminf _{\alpha \rightarrow 0}\left|\left\langle F g_{\alpha}, g_{\alpha}\right\rangle\right|<\infty$.

The assumptions required in this corollary are weaker than the ones required by the Factorization method but are similar to those of so-called inf-criterion (See [13]). Indeed the main advantage of GLSM with respect to the inf-criterion (as it will explained in the numerical section) is that it leads to a more tractable numerical inversion algorithms. In some special configurations there is a direct link between GLSM and the factorization method as explained below. 
We also remark that according to Lemma 2 the sequence $\left(g_{\alpha}\right)$ provides a nearby solution to $F g \simeq \phi$ satisfying

$$
\left\|F g_{\alpha}-\phi\right\| \leq j_{\alpha}(\phi)+C \alpha
$$

The reader then easily observe from the proof that one obtain the same conclusion in Corollary 1 if we replace the indicator function $\left|\left\langle F g_{\alpha}, g_{\alpha}\right\rangle\right|$ by $\left|\left\langle\phi, g_{\alpha}\right\rangle\right|$. The latter criterion coincides with the one proposed in [1] and has been analyzed in [1] and [2] based on the $\left(F^{*} F\right)^{\frac{1}{4}}$ method.

\subsection{Link with the $\left(F^{*} F\right)^{\frac{1}{4}}$ method}

We found it useful to indicate a link between the GLSM and the first version of the factorization method, namely the so-called $\left(F^{*} F\right)^{\frac{1}{4}}$-method [12]. This method applies when $X$ is a Hilbert space with a scalar product denoted (, ), and $F: X \rightarrow X$ is compact, normal, injective and with dense range. Then it is shown that $F$ can be factorized as

$$
F=\left(F^{*} F\right)^{\frac{1}{4}} J\left(F^{*} F\right)^{\frac{1}{4}}
$$

with $J: X \rightarrow X$ a coercive operator. Among others, two possibilities are of interest:

- A first possibility is to apply the GLSM with $B=F, H=\left(F^{*} F\right)^{\frac{1}{4}}$ and $G=$ $\left(F^{*} F\right)^{\frac{1}{4}} J$. We then obtain that $\phi \in \mathcal{R}\left(\left(F^{*} F\right)^{\frac{1}{4}}\right)$ if and only if $\limsup _{\alpha \rightarrow 0}\left|\left\langle F g_{\alpha}, g_{\alpha}\right\rangle\right|<$ $\infty$ where $g_{\alpha}$ satisfies $(9)$. Therefore, whenever one can use the range of $\left(F^{*} F\right)^{\frac{1}{4}}$ to characterize the shape of the scattering object, one can also use GLSM with $B=F$ to obtain a different characterization.

- Another (more informative) possibility is to apply GLSM with $B=\left(F^{*} F\right)^{\frac{1}{2}}$. In this case, using the system $\left(\lambda_{i}, \psi_{i}\right)_{i \geq 1}$ of eigenvalues and eigenvectors of the normal operator $F$, we observe that

$$
\begin{aligned}
J_{\alpha}(\phi ; g) & =\alpha\left|\left(\left(F^{*} F\right)^{\frac{1}{2}} g, g\right)\right|+\|F g-\phi\|^{2} \\
& =\alpha \sum_{i}\left|\lambda_{i}\right|\left|\left(g, \psi_{i}\right)\right|^{2}+\sum_{i}\left(\lambda_{i}\left(g, \psi_{i}\right)-\left(\phi, \psi_{i}\right)\right)^{2} .
\end{aligned}
$$

Hence, $J_{\alpha}(\phi ; \cdot)$ has a minimizer given by

$$
g_{\alpha}=\sum_{i} \frac{\overline{\lambda_{i}}\left(\phi, \psi_{i}\right)}{\alpha\left|\lambda_{i}\right|+\left|\lambda_{i}\right|^{2}} \psi_{i}
$$

It is clear that this $g_{\alpha}$ satisfies (9). Let us now define

$$
g_{\alpha}^{\mathrm{FM}}=\sum_{i} \frac{\left|\lambda_{i}\right|^{\frac{1}{2}}}{\left|\lambda_{i}\right|+\alpha}\left(\phi, \psi_{i}\right) \psi_{i}
$$

which is the minimizer of the Tikhonov functional $\alpha\|g\|^{2}+\left\|\left(F^{*} F\right)^{\frac{1}{4}} g-\phi\right\|^{2}$. Then one observes that the GLSM indicator is nothing but

$$
\left|\left(\left(F^{*} F\right)^{\frac{1}{2}} g_{\alpha}, g_{\alpha}\right)\right|=\sum_{i} \frac{\left|\lambda_{i}\right|\left(\phi, \psi_{i}\right)^{2}}{\left(\alpha+\left|\lambda_{i}\right|\right)^{2}}=\left\|g_{\alpha}^{\mathrm{FM}}\right\|^{2} .
$$


We finally remark that one obtains similar link with the so called $F_{\#}$ method (when it applies) and GLSM by taking $B=F_{\#}$ and replacing $F$ by $F_{\#}$ in the GLSM setting. But when the $F_{\#}$ method applies one can also apply GLSM with only $B=F$.

\subsection{Regularized formulation of GLSM}

As it will be clearer later, the previous formulation of GLSM has to be adapted to the case of noisy operators since in general a noisy operator $B$ does not satisfy a factorization of the form (5) (with a middle operator satisfying a coercivity property similar to (8)). In order to cope with this issue we introduce a regularized version of $J_{\alpha}$ which allows similar range characterization and where one both controls the noisy criteria and the noisy misfit term. Among several other options, it turned out that a convenient way to introduce this regularization is to consider for $\alpha>0$ and $\epsilon>0$ (that will later be linked with the noise level) and for $\phi \in X^{*}$, the functional $J_{\alpha}^{\varepsilon}(\phi ; \cdot): X \rightarrow \mathbb{R}$ defined by

$$
J_{\alpha}^{\varepsilon}(\phi ; g)=\alpha\left(|\langle B g, g\rangle|+\varepsilon\|g\|^{2}\right)+\|F g-\phi\|^{2} .
$$

Lemma 3. Assume that $B$ is compact. Then for all $\alpha>0, \epsilon>0$ and $\phi \in X^{*}$ the functional $J_{\alpha}^{\varepsilon}(\phi ; \cdot)$ has a minimizer $g_{\alpha}^{\varepsilon} \in X$. If we assume in addition that $F$ has dense range, then

$$
\lim _{\alpha \rightarrow 0} \lim _{\varepsilon \rightarrow 0} J_{\alpha}^{\varepsilon}\left(\phi ; g_{\alpha}^{\varepsilon}\right)=\lim _{\varepsilon \rightarrow 0} \limsup _{\alpha \rightarrow 0} J_{\alpha}^{\varepsilon}\left(\phi ; g_{\alpha}^{\varepsilon}\right)=0 .
$$

Proof. The existence of minimizer is clear: for fixed $\alpha>0, \epsilon>0$ and $\phi \in X^{*}$, any minimizing sequence $\left(g^{n}\right)$ of $J_{\alpha}^{\varepsilon}(\phi ; \cdot)$ is bounded and therefore one can assume that it is weakly convergent in $X$ to some $g_{\alpha}^{\varepsilon} \in X$. The lower semi-continuity of the norm with respect to weak convergence and the compactness property of $B$ then imply

$$
J_{\alpha}^{\varepsilon}\left(\phi ; g_{\alpha}^{\varepsilon}\right) \leq \liminf _{n \rightarrow \infty} J_{\alpha}^{\varepsilon}\left(\phi ; g^{n}\right) \leq \inf _{g \in X} J_{\alpha}^{\varepsilon}(\phi ; g)
$$

which proves that $g_{\alpha}^{\varepsilon}$ is a minimizer of $J_{\alpha}^{\varepsilon}(\phi ; \cdot)$ on $X$.

Now assume in addition that $F$ has dense range. By Lemma $2, j_{\alpha}(\phi) \rightarrow 0$ as $\alpha \rightarrow 0$. Showing that $\lim _{\varepsilon \rightarrow 0} J_{\alpha}^{\varepsilon}\left(\phi ; g_{\alpha}^{\varepsilon}\right)=j_{\alpha}(\phi)$ will then prove that $\lim _{\alpha \rightarrow 0} \lim _{\varepsilon \rightarrow 0} J_{\alpha}^{\varepsilon}\left(\phi ; g_{\alpha}^{\varepsilon}\right)=0$. We observe that

$$
J_{\alpha}^{\varepsilon}(\phi ; g)=J_{\alpha}(\phi ; g)+\alpha \varepsilon\|g\|^{2}
$$

and therefore $\left|J_{\alpha}^{\varepsilon}(\phi ; g)-J_{\alpha}(\phi ; g)\right| \rightarrow 0$ as $\varepsilon \rightarrow 0$. For $\eta>0$ one can choose $g$ such that $\left|J_{\alpha}(\phi ; g)-j_{\alpha}(\phi)\right| \leq \eta / 2$. For this $g$ one then has for $\varepsilon$ sufficiently small $\left|J_{\alpha}^{\varepsilon}(\phi ; g)-J_{\alpha}(\phi ; g)\right|<\eta / 2$. We obtain by triangular inequality that for $\varepsilon$ sufficiently small $J_{\alpha}^{\varepsilon}(\phi ; g) \leq j_{\alpha}(\phi)+\eta$. We now observe from the definitions of $g_{\alpha}^{\varepsilon}$ and $j_{\alpha}$ and from $(12)$

$$
j_{\alpha}(\phi) \leq J_{\alpha}\left(\phi ; g_{\alpha}^{\varepsilon}\right) \leq J_{\alpha}^{\varepsilon}\left(\phi ; g_{\alpha}^{\varepsilon}\right) \leq J_{\alpha}^{\varepsilon}(\phi ; g),
$$

which proves the claim.

We now prove $\lim _{\varepsilon \rightarrow 0} \limsup _{\alpha \rightarrow 0} J_{\alpha}^{\varepsilon}\left(\phi ; g_{\alpha}^{\varepsilon}\right)=0$. First consider $g_{\varepsilon}$ a minimizer on $X$ of the Tikhonov functional $\varepsilon^{2}\|g\|^{2}+\|F g-\phi\|^{2}$ and set $j^{\varepsilon}=\varepsilon^{2}\left\|g_{\varepsilon}\right\|^{2}+\left\|F g_{\varepsilon}-\phi\right\|^{2}$ 
which goes to zero as $\varepsilon$ goes to zero (classical result for Tikhonov regularization, see also Lemma 2 which is valid for any bounded operator $B$ ). We have that $\alpha \leq \varepsilon$, $J_{\alpha}^{\varepsilon}(g) \leq \varepsilon^{2}\|g\|^{2}+\|F g-\Phi\|^{2}+\alpha(|(B g, g)|$. For by taking the upper limit

$$
\limsup _{\alpha \rightarrow 0} J_{\alpha}^{\varepsilon}\left(g_{\alpha}^{\varepsilon}\right) \leq \limsup _{\alpha \rightarrow 0} J_{\alpha}^{\varepsilon}\left(g_{\varepsilon}\right)=j^{\varepsilon},
$$

which concludes the proof.

Theorem 4. Under the assumptions of Theorem 3 and the additional assumption that $B$ is compact the following holds. If $g_{\alpha}^{\varepsilon}$ denotes the minimizer of $J_{\alpha}^{\varepsilon}(\phi ; \cdot)$ (defined by (11)) for $\alpha>0, \varepsilon>0$ and $\phi \in X^{*}$, then $\phi \in \mathcal{R}(G)$ if and only if $\limsup _{\alpha \rightarrow 0} \limsup _{\varepsilon \rightarrow 0}\left|\left\langle B g_{\alpha}^{\varepsilon}, g_{\alpha}^{\varepsilon}\right\rangle\right|<$ $\infty$ which is true if and only if $\liminf _{\alpha \rightarrow 0} \liminf _{\varepsilon \rightarrow 0}\left|\left\langle B g_{\alpha}^{\varepsilon}, g_{\alpha}^{\varepsilon}\right\rangle\right|<\infty$.

Proof. The proof is similar to the proof of Theorem 3.

- Assume that $\phi=G(\varphi)$ for some $\varphi \in \overline{\mathcal{R}(H)}$. We consider the same $g_{0}$ as in the first part of the proof of Theorem 3 (that depends on $\alpha$ but is independent from $\varepsilon)$. Then we choose $\varepsilon$ such that $\varepsilon\left\|g_{0}\right\|^{2} \leq 1$. Then

$$
J_{\alpha}^{\varepsilon}\left(\phi ; g_{\alpha}^{\varepsilon}\right) \leq J_{\alpha}^{\varepsilon}\left(\phi ; g_{0}\right) \leq J_{\alpha}\left(\phi ; g_{0}\right)+\alpha
$$

Consequently

$$
\alpha\left|\left\langle B g_{\alpha}^{\varepsilon}, g_{\alpha}^{\varepsilon}\right\rangle\right| \leq J_{\alpha}^{\varepsilon}\left(\phi ; g_{\alpha}^{\varepsilon}\right) \leq \alpha+2 \alpha\|T\|\left(\alpha+\|\varphi\|^{2}\right)+\alpha\|G\|^{2}
$$

which proves $\limsup _{\alpha \rightarrow 0} \limsup _{\varepsilon \rightarrow 0}\left|\left\langle B g_{\alpha}^{\varepsilon}, g_{\alpha}^{\varepsilon}\right\rangle\right|<\infty$.

- Assume $\phi \notin \mathcal{R}(G)$ and assume that $\liminf _{\alpha \rightarrow 0} \liminf _{\varepsilon \rightarrow 0}\left|\left\langle B g_{\alpha}^{\varepsilon}, g_{\alpha}^{\varepsilon}\right\rangle\right|$ is finite. The coercivity of $T$ implies that $\liminf _{\alpha \rightarrow 0} \liminf _{\varepsilon \rightarrow 0}\left\|H g_{\alpha}^{\varepsilon}\right\|^{2}$ is also finite. This means the existence of a subsequence $\left(\alpha^{\prime}, \varepsilon\left(\alpha^{\prime}\right)\right)$ such that $\alpha^{\prime} \rightarrow 0$ and $\varepsilon\left(\alpha^{\prime}\right) \rightarrow 0$ as $\alpha^{\prime} \rightarrow 0$ and $\left\|H g_{\alpha^{\prime}}^{\varepsilon\left(\alpha^{\prime}\right)}\right\|^{2}$ is bounded independently from $\alpha^{\prime}$. On the other hand, the second part of Lemma 3 (namely the first limit), indicates that one can choose this subsequence such that $J_{\alpha^{\prime}}^{\varepsilon\left(\alpha^{\prime}\right)}\left(g_{\alpha^{\prime}}^{\varepsilon\left(\alpha^{\prime}\right)}\right) \rightarrow 0$ as $\alpha^{\prime} \rightarrow 0$ and therefore $\left\|F g_{\alpha^{\prime}}^{\varepsilon\left(\alpha^{\prime}\right)}-\phi\right\| \rightarrow 0$ as $\alpha^{\prime} \rightarrow 0$. The compactness of $G$ implies that a subsequence of $G H g_{\alpha^{\prime}}^{\varepsilon\left(\alpha^{\prime}\right)}$ converges for some $G \varphi$ in $X^{*}$. The uniqueness of the limit implies that $G \varphi=\phi$ which is a contradiction.

In this theorem $\varepsilon$ should be viewed as the regularization parameter (and not $\alpha$ which is rather used to construct an indicator function with a limiting process). As indicated by (13), this regularization parameter serves in the construction of the minimizing sequence of Theorem 3.

This theorem with regularization stipulates that a criterion to localize the target is given by $\left|\left\langle B g_{\alpha}^{\varepsilon}, g_{\alpha}^{\varepsilon}\right\rangle\right|$ for small values of $\epsilon$ and $\alpha$. The reader can easily see from the first part of the proof that the result holds true if we replace this by $\left(\left|\left\langle B g_{\alpha}^{\varepsilon}, g_{\alpha}^{\varepsilon}\right\rangle\right|+\varepsilon\left\|g_{\alpha}^{\varepsilon}\right\|^{2}\right)$. This latter criterion is more suited to the case of noisy measurements as indicated in the section below. 


\subsection{The GLSM for noisy data}

In this section we will consider the case where there may be noise in the data. More precisely, we shall assume that one has access to two noisy operators $B^{\delta}$ and $F^{\delta}$ such that

$$
\left\|F^{\delta}-F\right\| \leq \delta\|F\| \quad \text { and }\left\|B^{\delta}-B\right\| \leq \delta\|B\|
$$

for some $\delta>0$. We also assume in this section the operator, $B, B^{\delta} F^{\delta}$ and $F$ are compact. We then consider for $\alpha>0$ and $\phi \in X^{*}$, the functional $J_{\alpha}^{\delta}(\phi ; \cdot): X \rightarrow \mathbb{R}$,

$$
J_{\alpha}^{\delta}(\phi ; g):=\alpha\left(\left|\left\langle B^{\delta} g, g\right\rangle\right|+\delta\|B\|\|g\|^{2}\right)+\left\|F^{\delta} g-\phi\right\|^{2} \quad \forall g \in X,
$$

which coincides with a regularized noisy functional $J_{\alpha}^{\varepsilon}$ with a regularization parameter $\epsilon=\delta\|B\|$. According to Lemma 3 one can consider $g_{\alpha}^{\delta}$ a minimizer of $J_{\alpha}^{\delta}(\phi ; g)$. We first observe (similarly to in the second part of the proof of Lemma 3 )

Lemma 4. Assume in addition that $F$ has dense range. Then for all $\phi \in X^{*}$,

$$
\lim _{\alpha \rightarrow 0} \limsup _{\delta \rightarrow 0} J_{\alpha}^{\delta}\left(\phi ; g_{\alpha}^{\delta}\right)=0 .
$$

Proof. We observe that for all $g \in X$,

$$
J_{\alpha}^{\delta}(\phi ; g) \leq J_{\alpha}(\phi ; g)+\left(2 \alpha \delta\|B\|+\delta^{2}\|F\|^{2}\right)\|g\|^{2} .
$$

Since $\left(2 \alpha \delta\|B\|+\delta^{2}\|F\|^{2}\right) \rightarrow 0$ as $\delta \rightarrow 0$, then as in the proof of Lemma 3 , for any $\eta>0$ ( $\alpha$ fixed), one can choose $g \in X$ such that for sufficiently small $\delta$,

$$
J_{\alpha}^{\delta}(\phi ; g) \leq j_{\alpha}(\phi)+\eta
$$

Consequently, from the definition of $g_{\alpha}^{\delta}$,

$$
J_{\alpha}^{\delta}\left(g_{\alpha}^{\delta} ; \phi\right) \leq j_{\alpha}(\phi)+\eta
$$

This proves the claim, since $j_{\alpha}(\phi) \rightarrow 0$ as $\alpha \rightarrow 0$ (by Lemma 2).

Theorem 5. Assume that the assumptions of Theorem 3 and the additional assumptions of this subsection hold true. Let $g_{\alpha}^{\delta}$ be the minimizer of $J_{\alpha}^{\delta}(\phi ; \cdot)$ (defined by (21)) for $\alpha>0, \delta>0$ and $\phi \in X^{*}$. Then $\phi \in \mathcal{R}(G)$ if and only if $\limsup _{\alpha \rightarrow 0} \limsup _{\delta \rightarrow 0}\left(\left|\left\langle B^{\delta} g_{\alpha}^{\delta}, g_{\alpha}^{\delta}\right\rangle\right|+\delta\|B\|\left\|g_{\alpha}^{\delta}\right\|^{2}\right)<\infty$ which is true if and only if $\liminf _{\alpha \rightarrow 0} \liminf _{\delta \rightarrow 0}\left(\left|\left\langle B^{\delta} g_{\alpha}^{\delta}, g_{\alpha}^{\delta}\right\rangle\right|+\delta\|B\|\left\|g_{\alpha}^{\delta}\right\|^{2}\right)<\infty$.

Proof. The proof of this theorem follows the lines of the proof of Theorem 4.

- Assume that $\phi=G(\varphi)$ for some $\varphi \in \overline{\mathcal{R}(H)}$. We consider the same $g_{0}$ as in the first part of the proof of Theorem 3 (that depends on $\alpha$ but is independent from $\delta)$. Choosing $\delta$ sufficiently small such that

$$
\left(2 \alpha \delta\|B\|+\delta^{2}\|F\|^{2}\right)\left\|g_{0}\right\|^{2} \leq \alpha
$$


we get

$$
J_{\alpha}^{\delta}\left(\phi ; g_{\alpha}^{\delta}\right) \leq J_{\alpha}^{\delta}\left(\phi ; g_{0}\right) \leq J_{\alpha}\left(\phi ; g_{0}\right)+\alpha .
$$

Consequently

$$
\alpha\left(\left|\left\langle B g_{\alpha}^{\delta}, g_{\alpha}^{\delta}\right\rangle\right|+\delta\|B\|\left\|g_{\alpha}^{\delta}\right\|^{2}\right) \leq J_{\alpha}^{\delta}\left(\phi ; g_{\alpha}^{\delta}\right) \leq \alpha+2 \alpha\|T\|\left(\alpha+\|\varphi\|^{2}\right)+\alpha\|G\|^{2},
$$

which proves $\limsup _{\alpha \rightarrow 0} \limsup _{\delta \rightarrow 0}\left(\left|\left\langle B^{\delta} g_{\alpha}^{\delta}, g_{\alpha}^{\delta}\right\rangle\right|+\delta\|B\|\left\|g_{\alpha}^{\delta}\right\|^{2}\right)<\infty$.

- Assume $\phi \notin \mathcal{R}(G)$ and assume that $\liminf _{\alpha \rightarrow 0} \liminf _{\varepsilon \rightarrow 0}\left(\left|\left\langle B^{\delta} g_{\alpha}^{\delta}, g_{\alpha}^{\delta}\right\rangle\right|+\delta\|B\|\left\|g_{\alpha}^{\delta}\right\|^{2}\right)$ is finite. The coercivity of $T$ implies that

$$
\mu\left\|H g_{\alpha(\delta)}^{\delta}\right\|^{2} \leq\left|\left\langle B g_{\alpha}^{\delta}, g_{\alpha}^{\delta}\right\rangle\right| \leq\left|\left\langle B^{\delta} g_{\alpha}^{\delta}, g_{\alpha}^{\delta}\right\rangle\right|+\delta\|B\|\left\|g_{\alpha}^{\delta}\right\|^{2} .
$$

Therefore $\liminf _{\alpha \rightarrow 0} \liminf _{\delta \rightarrow 0}\left\|H g_{\alpha}^{\delta}\right\|^{2}$ is also finite. This means the existence of a subsequence $\left(\alpha^{\prime}, \delta\left(\alpha^{\prime}\right)\right)$ such that $\alpha^{\prime} \rightarrow 0$ and $\delta\left(\alpha^{\prime}\right) \rightarrow 0$ as $\alpha^{\prime} \rightarrow 0$ and $\left\|H g_{\alpha^{\prime}}^{\delta\left(\alpha^{\prime}\right)}\right\|^{2}$ is bounded independently from $\alpha^{\prime}$. One can also choose $\delta\left(\alpha^{\prime}\right)$ such that $\delta\left(\alpha^{\prime}\right) \leq \alpha^{\prime}$. On the other hand Lemma 4 indicates that one can choose this subsequence such that $J_{\alpha^{\prime}}^{\delta\left(\alpha^{\prime}\right)}\left(g_{\alpha^{\prime}}^{\delta\left(\alpha^{\prime}\right)}\right) \rightarrow 0$ as $\alpha^{\prime} \rightarrow 0$ and therefore $\left\|F^{\delta} g_{\alpha^{\prime}}^{\delta\left(\alpha^{\prime}\right)}-\phi\right\| \rightarrow 0$ as $\alpha^{\prime} \rightarrow 0$ and $\alpha^{\prime} \delta\left(\alpha^{\prime}\right)\left\|g_{\alpha^{\prime}}^{\delta\left(\alpha^{\prime}\right)}\right\|^{2} \rightarrow 0$ as $\alpha^{\prime} \rightarrow 0$. By a triangular inequality and $\delta\left(\alpha^{\prime}\right) \leq \alpha^{\prime}$ we then deduce that $\left\|F g_{\alpha^{\prime}}^{\delta\left(\alpha^{\prime}\right)}-\phi\right\| \rightarrow 0$ as $\alpha^{\prime} \rightarrow 0$. The compactness of $G$ implies that a subsequence of $G H g_{\alpha^{\prime}}^{\delta\left(\alpha^{\prime}\right)}$ converges for some $G \varphi$ in $X^{*}$. The uniqueness of the limit implies that $G \varphi=\phi$ which is a contradiction.

It is clear from the proof of the theorem that any strategy of regularization $\varepsilon(\delta)$ satisfying $\epsilon(\delta) \geq \delta\|B\|$ and $\epsilon(\delta) \rightarrow 0$ as $\delta \rightarrow 0$ would be convenient to obtain a similar result. From the numerical perspective this theorem indicates that a criterion to localize the object would be

$$
\left|\left\langle B^{\delta} g_{\alpha}^{\delta}, g_{\alpha}^{\delta}\right\rangle\right|+\delta\|B\|\left\|g_{\alpha}^{\delta}\right\|^{2}
$$

for small values of $\alpha$. Indeed the theorem only says that this criterion would be efficient for sufficiently small noise. Building explicit link between the value of $\alpha$ and the noise level $\delta$ (in the fashion of a posteriori regularization strategies) would be of valuable theoretical interest but this seems to be challenging (due to the compactness of the operator $B$ ). One can see from the proof that adding the term $\delta\|B\|\left\|g_{\alpha}^{\delta}\right\|^{2}$ is important to conclude when $\phi$ is not in the range of $G$. This means that this term is important for correcting the behavior of the indicator function outside the inclusion, which is corroborated by the numerical experiments below. 


\section{Some applications of GLSM}

We turn back to our model problem and consider the notation and assumptions of Section 2. We shall apply GLSM with $B=F$. The central additional theorem needed for this case is the following coercivity property of the operator $T$. This theorem holds true under the following assumptions on the refractive index.

Hypothesis 2. We assume that $n \in L^{\infty}\left(\mathbb{R}^{d}\right), \operatorname{supp}(n-1)=\bar{D}, \Im(n) \geq 0$ and there exists a constant $n_{0}>0$ such that $1-\Re(n(x))+\Im(n(x)) \geq n_{0}$ for a.e. $x \in D$ or $\Re(n(x))-1+\Im(n(x)) \geq n_{0}$ for a.e. $x \in D$.

We recall that the values of $k^{2} \in \mathbb{R}_{+}$for which Hypothesis 1 does not hold form a discrete set without finite accumulation point. The values $k^{2} \in \mathbb{R}_{+}$for which Hypothesis 1 does not hold will be referred in the sequel as transmission eigenvalues.

Theorem 6. Assume that Hypothesis 2 holds and that $k^{2} \in \mathbb{R}_{+}$is not a transmission eigenvalue. Then the operator $T$ defined by (4) satisfies the coercivity property (8) with $X=X^{*}=L^{2}(D)$ and the operator $H$ defined by (2).

Proof. For the reader convenience we start by proving a useful (classical) identity related to the imaginary part of $T$. With $($,$) denoting L^{2}(D)$ scalar product, for $\psi \in L^{2}(D)$ and $w \in H_{\text {loc }}^{1}\left(\mathbb{R}^{d}\right)$ solution of (1),

$$
(T \psi, \psi)=-k^{2} \int_{D}(1-n)(\psi+w) \bar{\psi} d x .
$$

We remark that by elliptic regularity, $w \in H_{\mathrm{loc}}^{2}\left(\mathbb{R}^{d}\right)$. Multiplying (1) with $\bar{w}$ and integrating by part over $B_{R}$ : a ball of radius $R$ containing $D$,

$$
k^{2} \int_{D}(1-n)(\psi+w) \bar{w} d x=-\int_{B_{R}}|\nabla w|^{2}-k^{2} n|w|^{2} d x+\int_{|x|=R} \frac{\partial w}{\partial r} \bar{w} d s .
$$

The Sommerfeld Radiation condition indicates that

$$
\lim _{R \rightarrow \infty} \Im \int_{|x|=R} \frac{\partial w}{\partial r} \bar{w} d s=k \int_{\mathbb{S}^{d-1}}\left|w^{\infty}\right|^{2} d s,
$$

Therefore, taking the imaginary part then letting $R \rightarrow \infty$ yields

$$
k^{2} \Im \int_{D}(1-n)(\psi+w) \bar{w} d x=\int_{D} k^{2} \Im(n)|w|^{2} d x+k \int_{\mathbb{S}^{d-1}}\left|w^{\infty}\right|^{2} d s .
$$

Consequently, decomposing $(\psi+w) \bar{\psi}=|\psi+w|^{2}-(\psi+w) \bar{w}$, we obtain the important identity,

$$
\Im(T \psi, \psi)=\int_{D} k^{2} \Im(n)\left(|\psi+w|^{2}+|w|^{2}\right) d x+k \int_{\mathbb{S}^{d-1}}\left|w^{\infty}\right|^{2} d s .
$$

We are now in position to prove the coercivity property using a contradiction argument. Assume for instance the existence of a sequence $\psi_{\ell} \in \mathcal{R}(H)$ such that

$$
\left\|\psi_{\ell}\right\|_{L^{2}(D)}=1 \quad \text { and }\left|\left(T \psi_{\ell}, \psi_{\ell}\right)\right| \rightarrow 0 \text { as } \ell \rightarrow \infty .
$$


We denote by $w_{\ell} \in H_{\text {loc }}^{2}\left(\mathbb{R}^{d}\right)$ solution of $(1)$ with $\psi=\psi_{\ell}$. Elliptic regularity implies that $\left\|w_{\ell}\right\|_{H^{2}(D)}$ is bounded uniformly with respect to $\ell$. Then up to changing the initial sequence, one can assume that $\psi_{\ell}$ weakly converges to some $\psi$ in $L^{2}(D)$ and $w_{\ell}$ converges weakly in $H_{\text {loc }}^{2}\left(\mathbb{R}^{d}\right)$ and strongly in $L^{2}(D)$ to some $w \in H_{\text {loc }}^{2}\left(\mathbb{R}^{d}\right)$. It is then easily seen (using distributional limit) that $w$ and $\psi$ satisfies (1), and since $\psi_{\ell} \in \mathcal{R}(H)$

$$
\Delta \psi+k^{2} \psi=0 \quad \text { in } D .
$$

Identity (18) and $\left|\left(T \psi_{\ell}, \psi_{\ell}\right)\right| \rightarrow 0$ implies that $w_{\ell}^{\infty} \rightarrow 0$ in $L^{2}\left(\mathbb{S}^{d-1}\right)$ and therefore $w^{\infty}=0$. The Rellich theorem and unique continuation principle implies $w=0$ outside $D$ and consequently $w \in H_{0}^{2}(D)$. With the help of equation (19) we get that $u=w+\psi \in L^{2}(D)$ and $v=\psi \in L^{2}(D)$ are such that $u-v \in H^{2}(D)$ and are solution of the interior transmission problem (3) with $f=g=0$. We then infer that $w=\psi=0$. Identity (17) applied to $\psi_{\ell}$ and $w_{\ell}$ implies

$$
\left|\left(T \psi_{\ell}, \psi_{\ell}\right)\right| \geq\left. k^{2}\left|\int_{D}(1-n)\right| \psi_{\ell}\right|^{2} d x\left|-k^{2}\right| \int_{D}(1-n) w_{\ell} \bar{\psi}_{\ell} d x \mid .
$$

Therefore, since $\int_{D}(1-n) w_{\ell} \bar{\psi}_{\ell} d x \rightarrow \int_{D}(1-n) w \bar{\psi} d x=0$, and using the assumptions on $n$,

$$
\lim _{\ell \rightarrow 0}\left|\left(T \psi_{\ell}, \psi_{\ell}\right)\right| \geq k^{2} n_{0} / 2>0
$$

which is a contradiction.

Let $C>0$ be a given constant (independent of $\alpha$ ) and consider for $\alpha>0$ and $z \in \mathbb{R}^{d}, g_{\alpha}^{z} \in L^{2}\left(\mathbb{S}^{d-1}\right)$ such that

$$
\alpha\left|\left(F g_{\alpha}^{z}, g_{\alpha}^{z}\right)\right|+\left\|F g_{\alpha}^{z}-\phi_{z}\right\|^{2} \leq j_{\alpha}\left(\phi_{z}\right)+C \alpha
$$

where

$$
j_{\alpha}\left(\phi_{z}\right)=\inf _{g \in L^{2}\left(\mathbb{S}^{d-1}\right)}\left(\alpha|(F g, g)|+\left\|F g-\phi_{z}\right\|^{2}\right) .
$$

Combining the results of Theorems 6 and 1 and the first claim of Theorem 2, we obtain the following as a straightforward application of Corollary 1.

Theorem 7. Assume that Hypothesis 2 holds and that $k^{2} \in \mathbb{R}_{+}$is not a transmission eigenvalue. Then $z \in D$ if and only if $\limsup _{\alpha \rightarrow 0}\left|\left(F g_{\alpha}^{z}, g_{\alpha}^{z}\right)\right|<\infty$ which is true if and only if $\liminf _{\alpha \rightarrow 0}\left|\left(F g_{\alpha}^{z}, g_{\alpha}^{z}\right)\right|<\infty$

For applications, it is important to rather use the criterion provided in Theorem 5. Consider $F^{\delta}: L^{2}\left(\mathbb{S}^{d-1}\right) \rightarrow L^{2}\left(\mathbb{S}^{d-1}\right)$ a compact operator such that

$$
\left\|F^{\delta}-F\right\| \leq \delta
$$

then consider for $\alpha>0$ and $\phi \in L^{2}\left(\mathbb{S}^{d-1}\right)$, the functional $J_{\alpha}^{\delta}(\phi ; \cdot): L^{2}\left(\mathbb{S}^{d-1}\right) \rightarrow \mathbb{R}$,

$$
J_{\alpha}^{\delta}(\phi ; g):=\alpha\left(\left|\left(F^{\delta} g, g\right)\right|+\delta\|g\|^{2}\right)+\left\|F^{\delta} g-\phi\right\|^{2} \quad \forall g \in L^{2}\left(\mathbb{S}^{d-1}\right) .
$$

Then as a direct consequence of Theorem 5 , we have the following characterization of $D$. 
Theorem 8. Assume that Hypothesis 2 holds and that $k^{2} \in \mathbb{R}_{+}$is not a transmission eigenvalue. For $z \in \mathbb{R}^{d}$ denote by $g_{\alpha, \delta}^{z}$ the minimizer of $J_{\alpha}^{\delta}\left(\phi_{z} ; \cdot\right)$ over $L^{2}\left(\mathbb{S}^{d-1}\right)$. Then

$z \in D$ if and only if $\limsup _{\alpha \rightarrow 0} \limsup _{\delta \rightarrow 0}\left(\left|\left(F^{\delta} g_{\alpha, \delta}^{z}, g_{\alpha, \delta}^{z}\right)\right|+\delta\left\|g_{\alpha, \delta}^{z}\right\|^{2}\right)<\infty$ which is true if and only if $\liminf _{\alpha \rightarrow 0} \liminf _{\delta \rightarrow 0}\left(\left|\left(F^{\delta} g_{\alpha, \delta}^{z}, g_{\alpha, \delta}^{\delta}\right)\right|+\delta\left\|g_{\alpha, \delta}^{z}\right\|^{2}\right)<\infty$.

The numerical algorithm associated with this theorem is given in next section. Let us note again as conclusion of this section that the results of Theorems 7 and 8 in fact apply whenever the so called $F_{\#}$ method apply. For instance the result hold true for obstacle scattering with Dirichlet boundary conditions, Neumann boundary conditions or impedance boundary conditions $[13,5]$. One has just to remove assumption 2 and instead of excluding transmission eigenvalues, one has to exclude the resonant eigenfrequencies associated with the interior problem. One can also apply GLSM to cracks as a consequence of the work in [3]. For Maxwell's equations one can in principle also treat the inverse medium problem but the GLSM method does allow to treat (in its current form) the case of inverse obstacle scattering (i.e. for instance perfectly or imperfectly conducting obstacles).

\section{Numerical algorithms issued from GLSM and validation}

Minimizing $J_{\alpha}^{\delta}$ (defined in equation 21) with $B=F$ may be computationally expensive and not straightforward (see Section 5.2). Thus we first propose to use the indicator function of the GLSM with the solution of the LSM, which can be seen as a generalisation of [2] in the case of noisy measurement. Then we introduce a second algorithm which is a post processing in the sense that it uses the solution of the LSM both to initialise the optimisation algorithm that minimize $J_{\alpha}^{\delta}$ and to initialise the parameter $\alpha$.

In order to fix the ideas, we shall restric ourselves to the two dimensional case and will introduce the algorithms for the discrete version of GLSM. We identify $\mathbb{S}^{1}$ with the interval $[0,2 \pi$. In order to collect the data of the inverse problem we solve numerically (1) for $\mathrm{N}$ incident fields $u^{i}\left(\frac{2 \pi j}{\mathrm{~N}}, \cdot\right), j \in\{0 \ldots \mathrm{N}-1\}$ using the surface integral equation forward solver available in [10]. The discret version of $F$ is then the matrix $F_{\mathrm{N}}:=\left(u^{\infty}\left(\frac{2 \pi j}{\mathrm{~N}}, \frac{2 \pi k}{\mathrm{~N}}\right)\right)_{0 \leq j, k \leq \mathrm{N}-1}$. We add some noise to the data to build a noisy far field matrix $F_{N}^{\delta}$ where $\left(F_{\mathrm{N}}^{\delta}\right)_{j, k}=\left(F_{\mathrm{N}}\right)_{j, k}\left(1+\sigma N_{i j}\right)$ for $\sigma>0$ and $N_{i j}$ an uniform complex random variable in $[-1,1]^{2}$. We denote $\Phi_{z, \mathrm{~N}} \in \mathbb{C}^{\mathrm{N}}$, the vector defined by $\Phi_{z, \mathrm{~N}}(j)=\phi_{z}\left(\frac{2 \pi j}{\mathrm{~N}}\right)$ for $0 \leq j \leq \mathrm{N}-1$.

\subsection{The use of GLSM as a new indicator function for the $L S M$}

We introduce the Tikhonov regularized solution of the far field equation

$$
g_{z, \mathrm{~N}}^{\eta, \mathrm{LSM}}:=\operatorname{argmin}_{g_{\mathrm{N}}} \eta\left\|g_{\mathrm{N}}\right\|_{L^{2}\left(\mathbb{S}^{1}\right)}^{2}+\left\|F_{\mathrm{N}}^{\delta} g_{\mathrm{N}}-\Phi_{z, \mathrm{~N}}\right\|_{L^{2}\left(\mathbb{S}^{1}\right)}^{2},
$$


where the regularization parameter $\eta$ is chosen using the Morozov discrepancy principle, i.e. $\eta$ is defined as the unique solution of

$$
\left\|F_{\mathrm{N}}^{\delta} g_{z, \mathrm{~N}}^{\eta, \mathrm{LSM}}-\Phi_{z, \mathrm{~N}}\right\|_{L^{2}\left(\mathbb{S}^{1}\right)}=\delta\left\|g_{z, \mathrm{~N}}^{\eta, \mathrm{LSM}}\right\|_{L^{2}\left(\mathbb{S}^{1}\right)} .
$$

Solving the same two equations with $F_{\#}^{\frac{1}{2}}$ or $\left(F^{*} F\right)^{\frac{1}{4}}$, depending of the nature of the scatter, instead of $F$ will give the solution of the factorization method $g_{z, \mathrm{~N}}^{\eta, \mathrm{FM}}$. To solve both the LSM and the FM equations we rely on the singular value decomposition of $F_{\mathrm{N}}^{\delta}$, which gives an explicit solution like in 3.2 .

As proposed in [2],[12] and [7], from these two problems three indicator functions can be computed:

$$
\begin{aligned}
& \mathcal{I}^{\mathrm{LSM}}(z)=\frac{1}{\left\|g_{z, \mathrm{~N}}^{\eta, \mathrm{LSM}}\right\|_{L^{2}\left(\mathbb{S}^{1}\right)}} \\
& \mathcal{I}^{\mathrm{HLSM}}(z)=\frac{1}{\sqrt{\left|H g_{z, \mathrm{~N}}^{\eta, \mathrm{LSM}}(z)\right|}}=\frac{1}{\sqrt{\mid\left(\Phi_{z, \mathrm{~N}}, g_{z, \mathrm{~N}}^{\eta, \mathrm{LSM}}\right)_{L^{2}\left(\mathbb{S}^{1}\right)}}} \\
& \mathcal{I}^{\mathrm{FM}}(z)=\frac{1}{\left\|g_{z, \mathrm{~N}}^{\eta, \mathrm{FM}}\right\|_{L^{2}\left(\mathbb{S}^{1}\right)}}
\end{aligned}
$$

As shown in the previous sections, a fourth indicator function is relevant, namely

$$
\mathcal{I}^{\mathrm{GLSM}}(z)=\frac{1}{\sqrt{\left|\left(F_{\mathrm{N}}^{\delta} g_{z, \mathrm{~N}}^{\eta, \mathrm{LSM}}, g_{z, \mathrm{~N}}^{\eta, \mathrm{LSM}}\right)\right|_{L^{2}\left(\mathbb{S}^{1}\right)}+\delta\left\|g_{z, \mathrm{~N}}^{\eta, \mathrm{LSM}}\right\|_{L^{2}\left(\mathbb{S}^{1}\right)}^{2}}}
$$

This indicator is indeed motivated by GLSM. However let us note that since $g_{z, \mathrm{~N}}^{\eta, \mathrm{LSM}}$ is not the minimizer of $J_{\alpha}^{\delta}(\phi ; \cdot)$ (defined in equation $(21)$ ) the theory developed here does not apply for this indicator function (a last indicator function covered by the theory will be build in section 5.2 using a more computationally complex method). The numerical experiments presented below indicate in the same time that this indicator function provide results comparable to the Factorization method.

We will present two simulations: one where two ellipses have Dirichlet boundary conditions and one where $n=2+0.5 i$ in one ellipse and $2+0.1 i$ in the other. In both examples $\mathrm{N}=100$ and we will consider $\frac{\left\|F_{\mathrm{N}}^{\delta}-F_{\mathrm{N}}\right\|}{\left\|F_{\mathrm{N}}\right\|}=0,1$ and $5 \%$.

Figures 1 and 2 show the results of the four indicator functions. First we see that $\mathcal{I}^{\text {HLSM }}$ is not robust to noise, the area outside the obstacle shows artefact where the indicator function is greater than inside the obstacle. This is an expected result since as stated at the end of 3.1 one can easily replace $\left|\left\langle F g_{\alpha}, g_{\alpha}\right\rangle\right|$ by $\left|\left\langle\phi, g_{\alpha}\right\rangle\right|$, which is not a valid indicator function in the presence of noise. Finally $\mathcal{I}^{\mathrm{LSM}}$ recover with less precision the border of the shape than $\mathcal{I}^{\mathrm{FM}}$ and $\mathcal{I}^{\mathrm{GLSM}}$ which exhibit comparable results. 

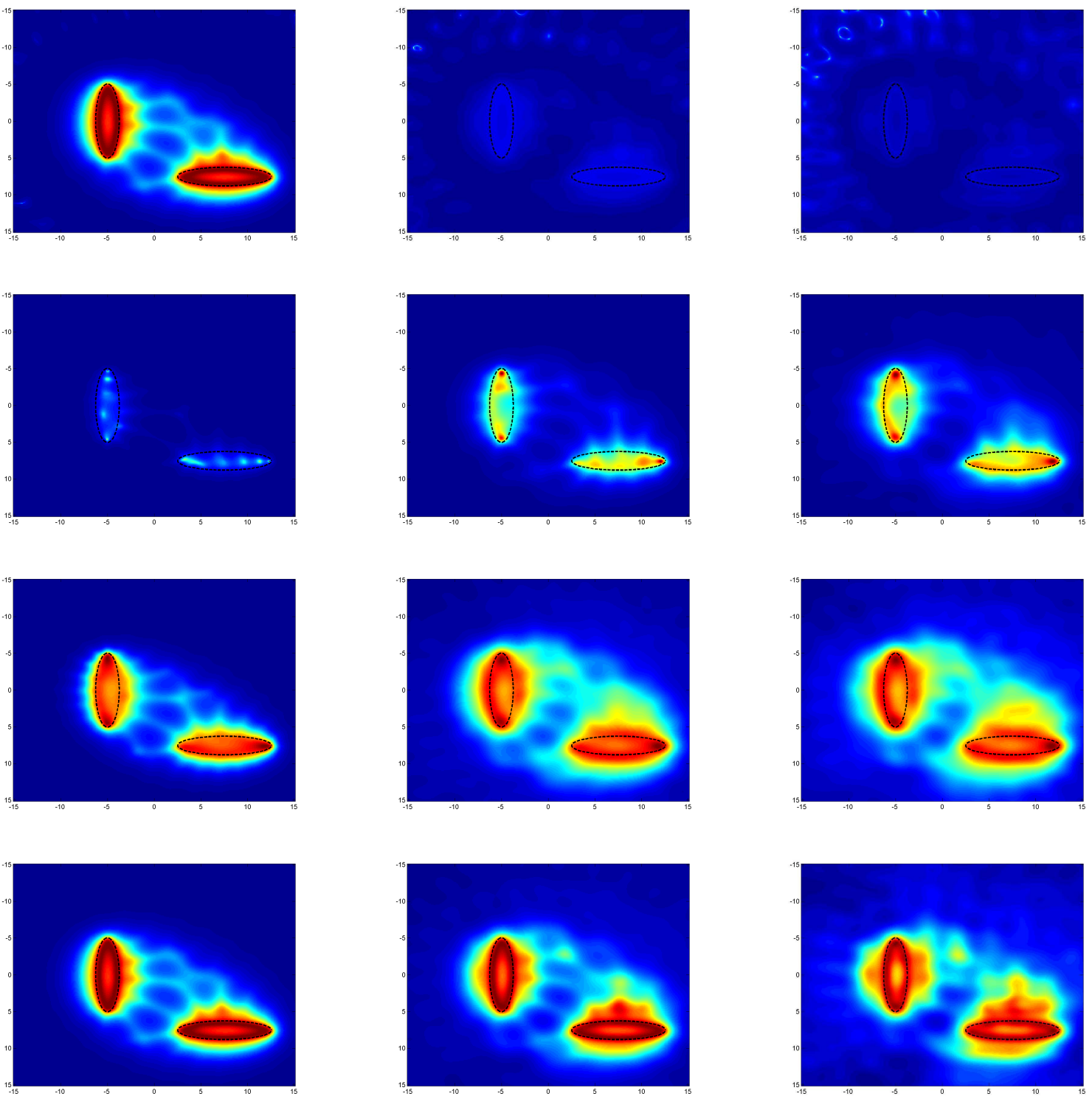

Figure 1. $\mathcal{I}^{\mathrm{HLSM}}$ (first line), $\mathcal{I}^{\mathrm{LSM}}$ (second line), $\mathcal{I}^{\mathrm{FM}}$ (third line) and $\mathcal{I}^{G L S M}$ (forth line) applied to the Dirichlet scatters for 0,1 and $5 \%$ of noise (from left to right)

\section{2. minimizing $J_{\alpha}^{\delta}$ : a post-processing}

In order to apply Theorem 8, we should find the minimizer of $J_{\alpha}^{\delta}(\phi ; \cdot)$ (defined in equation (21)). There are two main difficulties in this theorem. First, we do not have an analytic solution of the minimizer thus we will rely on an optimisation algorithm and as already mentioned in 3.4 and second we do not have an a priori method to link $\alpha$ to the noise level. Because of the good performance of the Morozov discrepancy principle we look for an heuristic that stay close to this principle. Since we have 

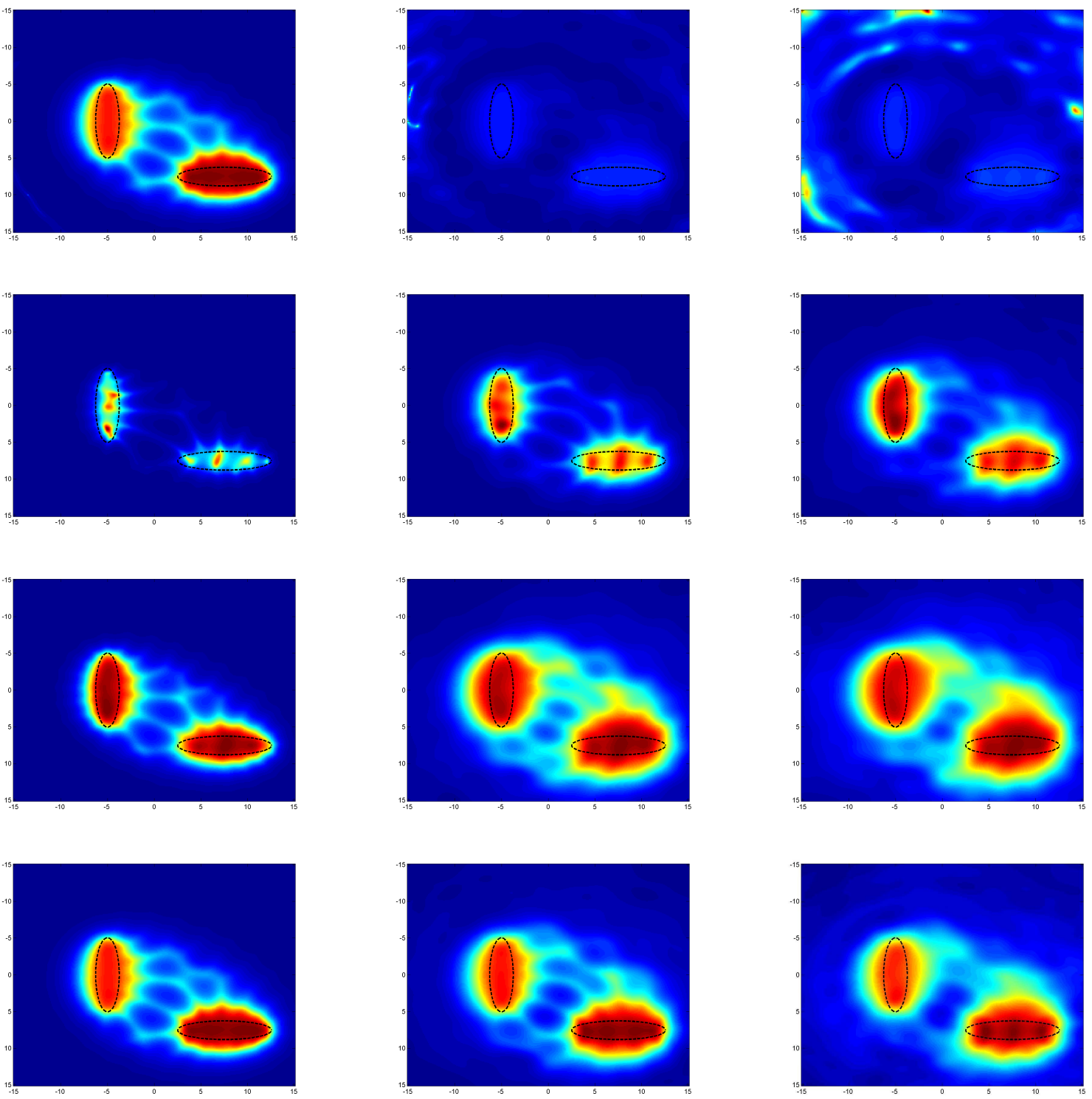

Figure 2. $\mathcal{I}^{\mathrm{HLSM}}$ (first line), $\mathcal{I}^{\mathrm{LSM}}$ (second line), $\mathcal{I}^{\mathrm{FM}}$ (third line) and $\mathcal{I}^{\mathrm{GLSM}}$ (forth line) applied to penetrable scatters for 0,1 and $5 \%$ of noise (from left to right))

$$
\begin{gathered}
\alpha\left(\left|\left(F^{\delta} g, g\right)\right|+\delta\|g\|^{2}\right) \leq \alpha\left(\left\|F^{\delta}\right\|+\delta\right)\|g\|^{2}, \text { we choose: } \\
\alpha=\frac{\eta_{\mathrm{LSM}}}{\left\|F^{\delta}\right\|+\delta}
\end{gathered}
$$

where $\eta_{\text {LSM }}$ is the parameter found when one applies the Morozov discrepancy principle to the Tikhonov formulation of the LSM.

Remark. The inequality $\left|\left(F^{\delta} g, g\right)\right| \leq\left\|F^{\delta}\right\|\|g\|^{2}$, we use to find the previous heuristic will reduce the strength of the penalty term compared to the Tikhonov-LSM. Moreover the fact that this inequality is coarser for eigenvector corresponding to small eigenvalue, means that the penality term will be smaller for points outside the obstacle. This is 
shown by figure 3, where we see that after the optimisation process the solution deviates from the Morozov discrepancy principles mainly outside the obstacle.

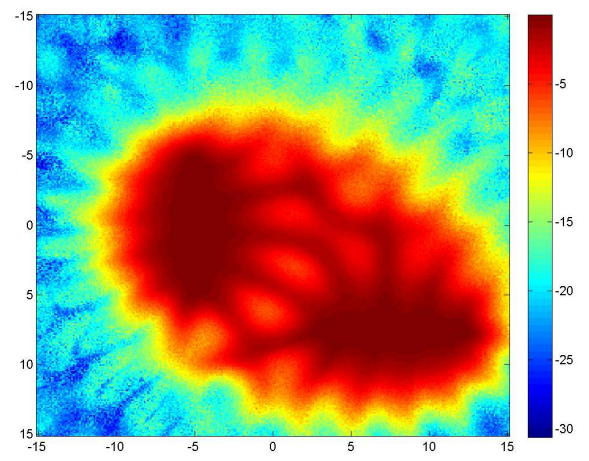

Figure 3. $\left\|F_{N}^{\delta} g_{\mathrm{N}}-\Phi_{z, \mathrm{~N}}\right\|-\delta\left\|g_{\mathrm{N}}\right\|$ after minimisation on the Dirichlet scatters with $5 \%$ of noise.

Minimizing $J_{\alpha}^{\delta}(\phi ; \cdot)$ in $\mathbb{C}^{\mathrm{N}}$ is not an easy task since it is a not differentiable nor a convex cost functionnal. However we can hope that $g_{z, \mathrm{~N}}^{\eta, \mathrm{LSM}}$ will be close to a minimum which makes it worth to try a gradient method. As explained in [16] gradient method extended well for complex variable if one look at $J_{\alpha}^{\delta}\left(\phi ; g_{\mathrm{N}}\right)$ as a function of two variables, $g_{\mathrm{N}}$ and $\bar{g}_{\mathrm{N}}$, knowing that one can compute the gradient of $J_{\alpha}^{\delta}$ with respect to $g_{\mathrm{N}}$ :

$$
\nabla_{\bar{g}_{\mathrm{N}}} J_{\alpha}^{\delta}\left(\phi ; g_{\mathrm{N}}, \bar{g}_{\mathrm{N}}\right):=\alpha\left(\frac{F_{\mathrm{N}}^{\delta} g_{\mathrm{N}} \cdot \bar{g}_{\mathrm{N}}}{\left|F_{\mathrm{N}}^{\delta} g_{\mathrm{N}} \cdot \bar{g}_{\mathrm{N}}\right|} F_{\mathrm{N}}^{\delta} g_{\mathrm{N}}+\delta g_{\mathrm{N}}\right)+F_{\mathrm{N}}^{\delta *}\left(F_{\mathrm{N}}^{\delta} g_{\mathrm{N}}-\Phi_{\mathrm{N}}\right)
$$

where $\cdot$ is the standard scalar product between vector. We did not change the absolute value with a differentiable surrogate because with the initial guess we used it was not necessary, this is supported by the fact that for the unperturbed operator $F$ the coercivity implies that $|(F g, g)|$ is never zero when $g$ is not zero.

Finally to do the optimisation we use the non-linear conjugate gradient implemented in [17] with a modified Hestenes-Stiefel heuristic to update the direction descent, which is described in algorithm 1 . We choose drastic stopping rules in order to ensure the convergence of the algorithm however we observe that convergence occurs before those stopping rules are satisfied. The design of a tailored method and set of parameters to minimize $J_{\alpha}^{\delta}$ would be an interesting perspective for this work.

The result of this optimisation performed for each $z$, gives us a new set: $g_{z, N}^{\alpha, \mathrm{GLSM}}$ which ultimately creates a new indicator function:

$$
\mathcal{I}^{\text {GLSMoptim }}=\frac{1}{\sqrt{\left|\left(F_{\mathrm{N}}^{\delta} g_{z, \mathrm{~N}}^{\alpha, \mathrm{GLSM}}, g_{z, \mathrm{~N}}^{\alpha, \mathrm{GLSM}}\right)\right|+\delta\left\|g_{z, N}^{\alpha, \mathrm{GLSM}}\right\|^{2}}}
$$

Figures 4 and 5 show that this post processing increases the quality of the reconstruction especially in the space in-between the two scatters. Moreover figure 6 shows that the improvement on an isolated scattered, a kite of contrast $n=2+0.5 i$, is less impressive (i.e. we do not improve the reconstruction of the non-convex part of the kite). 


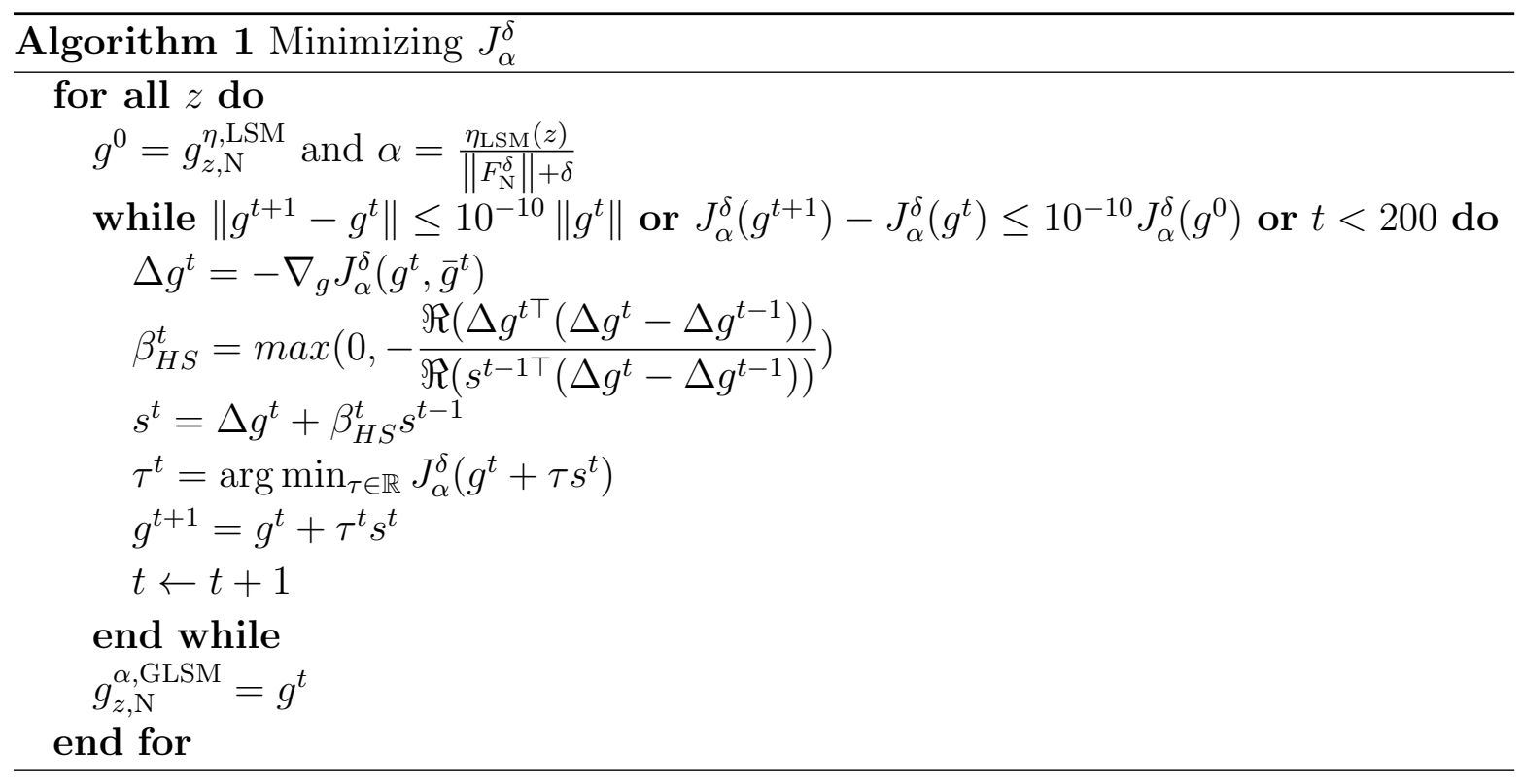

Remark. In the (less general) case where the $F_{\#}$ method is valid, one could choose $B^{\delta}=F_{\#}^{\delta}=\left|\Re\left(F^{\delta}\right)\right|+\left|\Im\left(F^{\delta}\right)\right|$ in equation (21). We know that $F_{\#}^{\delta}$ is a positive and self-adjoint operator then one can drop the absolute value in the definition of $J_{\alpha}^{\delta}$ :

$$
J_{\alpha}^{\delta}(\phi ; g):=\alpha\left(\left\|\left(F_{\#}^{\delta}\right)^{\frac{1}{2}} g\right\|^{2}+\delta_{\#}\|g\|^{2}\right)+\left\|F^{\delta} g-\phi\right\|^{2}
$$

and find $g_{z, \mathrm{~N}}^{\alpha, \mathrm{GLSM}}$ easily by solving the following (iteration-free) problem:

$$
\alpha\left(\left(F_{\#}^{\delta}\right)^{\frac{1}{2} *}\left(F_{\#}^{\delta}\right)^{\frac{1}{2}} g_{N}+\delta_{\#} g_{N}\right)+F^{\delta *}\left(F^{\delta} g_{N}-\Phi_{N}\right)=0
$$

\section{References}

[1] Tilo Arens. Why linear sampling works. Inverse Problems, 20(1):163-173, 2004.

[2] Tilo Arens and Armin Lechleiter. The linear sampling method revisited. J. Integral Equations Appl., 21(2):179-202, 2009.

[3] Yosra Boukari and Houssem Haddar. The Factorization method applied to cracks with impedance boundary conditions. Inverse Problems and Imaging, 2013. To appear.

[4] Fioralba Cakoni and David Colton. Qualitative methods in inverse scattering theory. Interaction of Mechanics and Mathematics. Springer-Verlag, Berlin, 2006. An introduction.

[5] Mathieu Chamaillard, Nicolas Chaulet, and Houssem Haddar. Analysis of the factorization method for a general class of boundary conditions. 2013. Preprint.

[6] David Colton, Houssem Haddar, and Michele Piana. The linear sampling method in inverse electromagnetic scattering theory. Inverse Problems, 19(6):S105-S137, 2003. Special section on imaging.

[7] David Colton and Andreas Kirsch. A simple method for solving inverse scattering problems in the resonance region. Inverse Problems, 12(4):383-393, 1996.

[8] David Colton and Rainer Kress. Inverse acoustic and electromagnetic scattering theory, volume 93 of Applied Mathematical Sciences. Springer, New York, third edition, 2013.

[9] Klaus Erhard and Roland Potthast. A numerical study of the probe method. SIAM J. Sci. Comput., 28(5):1597-1612, 2006.

[10] H. Haddar. Sampling 2d, Mars 2013. http://sourceforge.net/projects/samplings-2d/. 

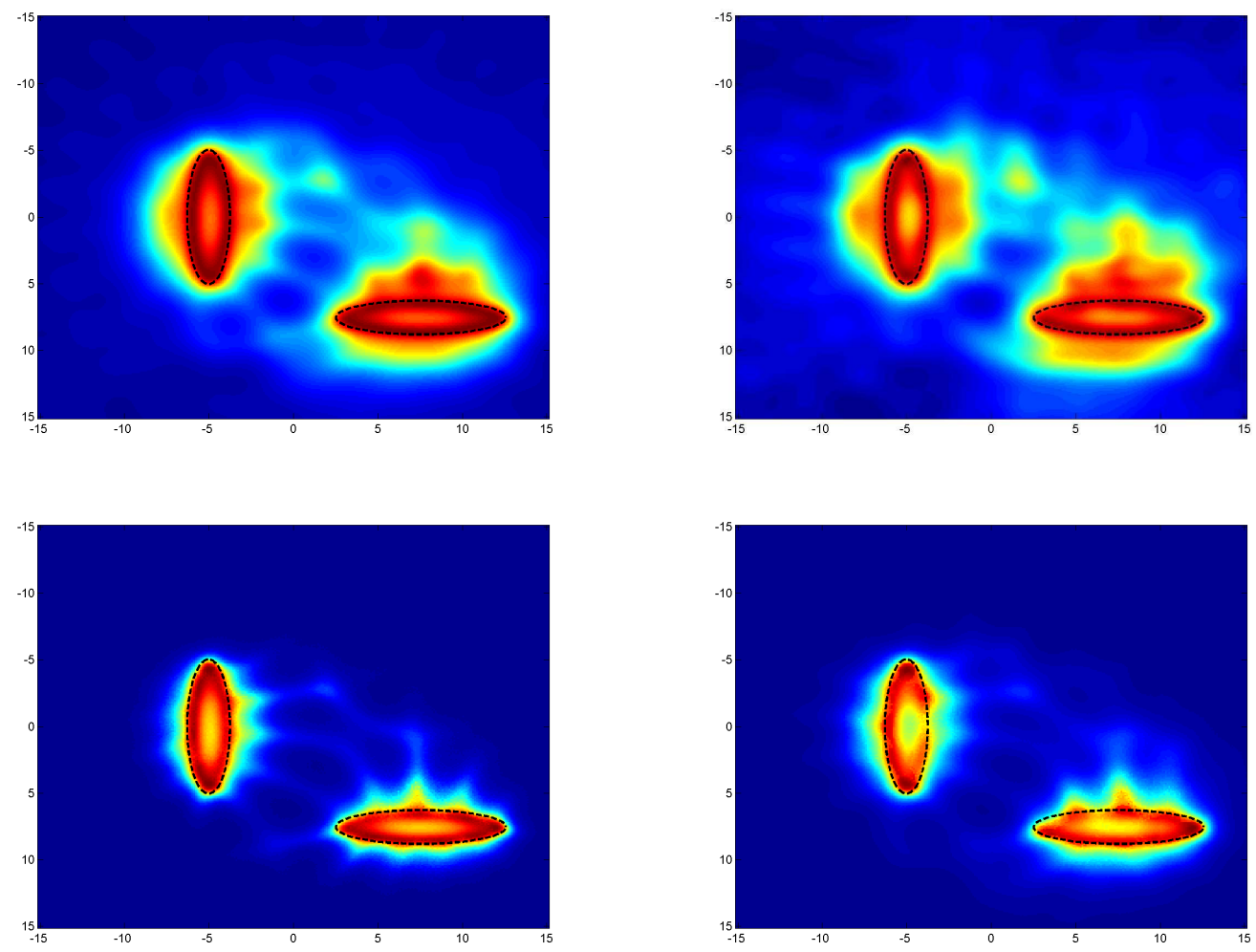

Figure 4. $\mathcal{I}^{\mathrm{GLSM}}$ (first line) and $\mathcal{I}^{\text {GLSMoptim }}$ (second line) applied to the Dirichlet scatters for 1 and $5 \%$ of noise (from left to right)

[11] Masaru Ikehata. A new formulation of the probe method and related problems. Inverse Problems, 21(1):413-426, 2005.

[12] Andreas Kirsch. Characterization of the shape of a scattering obstacle using the spectral data of the far field operator. Inverse Problems, 14(6):1489-1512, 1998.

[13] Andreas Kirsch and Natalia Grinberg. The factorization method for inverse problems, volume 36 of Oxford Lecture Series in Mathematics and its Applications. Oxford University Press, Oxford, 2008.

[14] Adrian I. Nachman, Lassi Päivärinta, and Ari Teirilä. On imaging obstacles inside inhomogeneous media. J. Funct. Anal., 252(2):490-516, 2007.

[15] B. P. Rynne and B. D. Sleeman. The interior transmission problem and inverse scattering from inhomogeneous media. SIAM J. Math. Anal., 22(6):1755-1762, 1991.

[16] L. Sorber, M. Barel, and L. Lathauwer. Unconstrained optimization of real functions in complex variables. SIAM J. Optim., 22(3):879-898, 2012.

[17] L. Sorber, M. Barel, and L. Lathauwer. Complex optimization toolbox v1.0, February 2013. http://esat.kuleuven.be/sista/cot/.

[18] John Sylvester. Discreteness of transmission eigenvalues via upper triangular compact operators. SIAM J. Math. Anal., 44(1):341-354, 2012. 

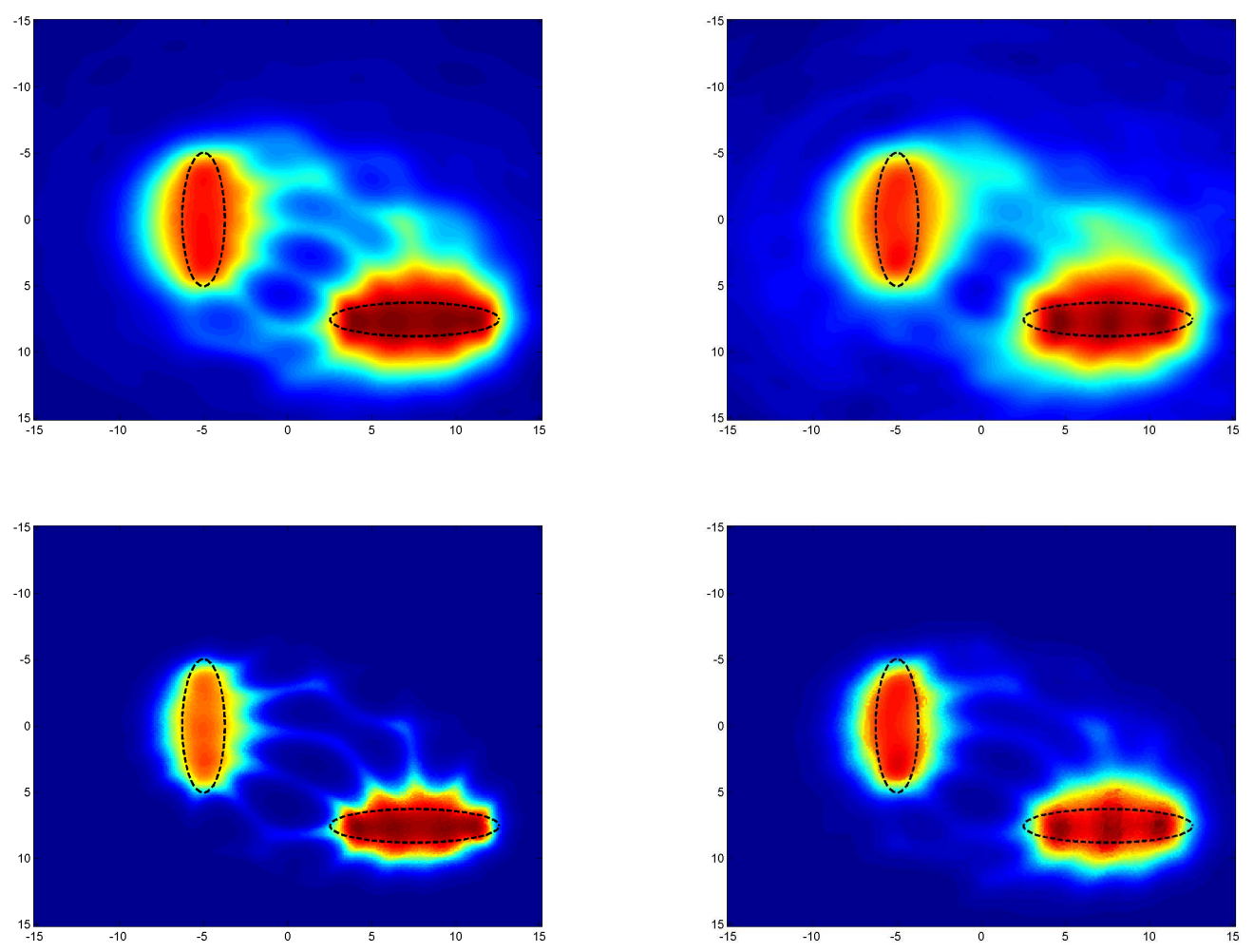

Figure 5. $\mathcal{I}^{\mathrm{GLSM}}$ (first line) and $\mathcal{I}^{\mathrm{GLSMoptim}}$ (second line) applied to the penetrable scatters for 1 and $5 \%$ of noise (from left to right)
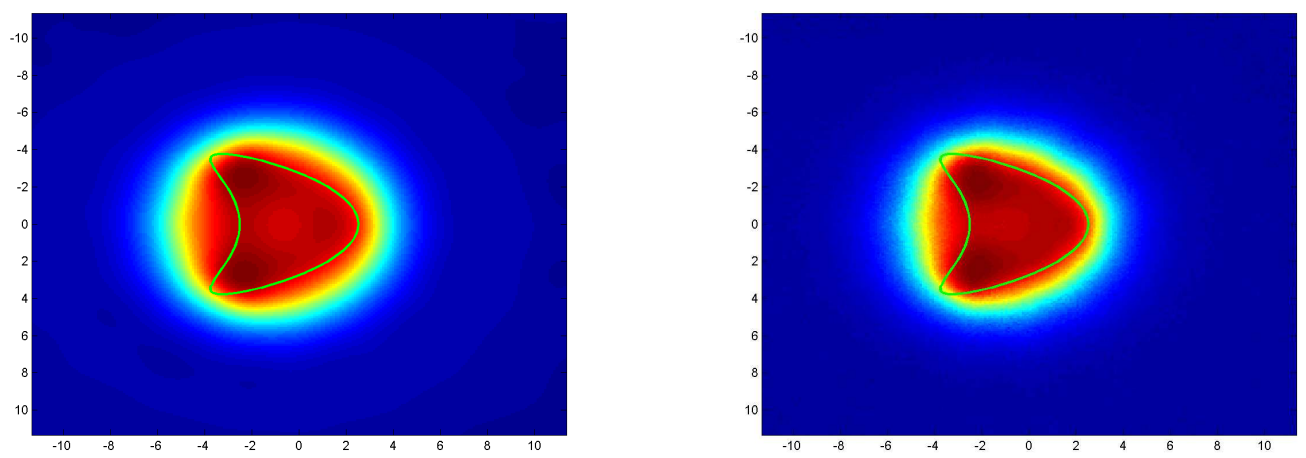

Figure 6. $\mathcal{I}^{\mathrm{GLSM}}$ and $\mathcal{I}^{\text {GLSMoptim }}$ (from left to right) with $1 \%$ of noise 